\title{
GLOBALEDU - AN ARCHITECTURE TO SUPPORT LEARNING IN A PERVASIVE COMPUTING ENVIRONMENT
}

\author{
Débora N. F. Barbosa ${ }^{1,2}$, Cláudio F. R. Geyer ${ }^{2}$ and Jorge L. V. Barbosa ${ }^{3}$ \\ ${ }^{1}$ Computer Science Course - Unilasalle University (UNILASALLE) - Av.Victor Barreto, 2.288 \\ - Canoas - RS - Brazil - nice(Ounilasalle.edu.br; \\ ${ }^{2}$ Institute of Informatics - Federal University of Rio Grande do Sul (UFRGS) Av. Bento \\ Gonçalves, 9500 - Bloco IV - Porto Alegre - RS - Brazil - gever@inf.ufrgs.br;
}

${ }^{3}$ PIPCA - University of Vale do Rio dos Sinos (UNISINOS) - Av. Unisinos, 950 - Bloco B São Leopoldo - RS - Brazil -jbarbosa@unisinos.br.

\begin{abstract}
This paper presents a propose for support learning in a pervasive computing environment. It is composed of educational service agents (ESA) and a pervasive personal pedagogical agent (P3A). ESA will support educational applications using the pervasive environment ISAM. Agents will be accessed through $\mathrm{P} 3 \mathrm{~A}$, which shall always be with the learner (the agent moves to devices that the learner is using - desktop, cellular phone, handhelds, etc), assisting the process of global learning through the identification and adaptation of the resources in agreement with the learner's educational model composed of the learner model, learner context and learning object model.
\end{abstract}

Keywords: Pervasive Learning, Pervasive Personal Pedagogical Agent, Learning Services Agent 


\section{INTRODUCTION}

The increasing use of mobile devices (mobile computing), the Internet, and wireless network designs a scenario where computing will be global. The mobility of the user requires new models of applications for computational power spread all over the net, nonresident in a device that has the sporadical capacity of communication, and that stores and executes software. This is the context of pervasive computing ${ }^{13}$. This is a scenario where the user is free to move anywhere, anytime, maintaining the network access and having access to his/her virtual computing environment. The essence is that the user's applications are available in a suitable adapted form, wherever that user goes. This category includes location-aware application, whose behavior is determined by the physical position of the user/device. In mobile computing environments, location has a profound effect. The current location of the user determines its current context; thus, location is an element of the application context.

In the global connectivity scenario, new learning and computational environments are essential, because the relationship between teaching and learning changes ${ }^{3}$. In our point of view, educational resources are distributed in the global network and the learning process connects them. Educational applications will allow all the context of the learner to be connected with the educational context, joining current information (for example, a work of art in a museum that is being visited) with educational objectives (the learner can be studying history of art or a painting).

Pervasive Computing is interesting for the development of learning applications because learning can occur anywhere, anytime, with continued computing support. For us, it is a Pervasive Learning perspective. Towards this scenario, some proposals are being developed, for example ${ }^{2-4,9,14}$.

In this paper, we describe a learning architecture called GlobalEdu. It is composed of educational service agents (ESA) and a pervasive personal pedagogical agent (P3A). ESA will support educational applications using the pervasive environment ISAM ${ }^{1,16,17}$. Agents will be accessed through $\mathrm{P} 3 \mathrm{~A}$, which shall be always with the learner, assisting the process of global education through the identification and adaptation of the resources in agreement with the learner's educational model composed of the learner model, learner context and learning object model.

This paper is organized as follows: Section 2 presents the definitions and standards associated with this work. Section 3 describes our architecture and the pedagogical agent used at this work. Section 4 concludes the paper and presents the plans for future works. 


\section{BACKGROUND}

Mobile learning is fundamentally about increasing learners' capability to physically move their own learning environment with them. Mobile learning is implemented with lightweight devices such as PDA, cellular mobile phones, and so on. Those mobile devices can connect to the Internet with wireless communication technologies, and enable learning at anytime and anywhere. In this situation, however, computers are not embedded in learner's surrounding environment, and they cannot seamlessly and flexibly obtain information about the context of his/her learning. Works such as ${ }^{9,11,15}$ have used mobile learning technology.

In pervasive learning, computers can obtain information about the learning context from the learning environment where small devices such as sensors, pads, badges, and so on, are embedded and communicate mutually. A pervasive learning environment can be built either by embedding models of specific environment into dedicated computers, or by building generic capabilities into computers to inquire, detect, explore, and dynamically build models of their environments. However, this makes availability and usefulness of pervasive learning limited and highly localized.

Our work does not consider embedded computers in the environment. We consider that environment systems support distributed, mobile, contextaware, adaptative and follow-me learning applications. Thus, we work in a pervasive learning perspective. Considering a pervasive perspective and based on several works, one of the characteristics of learning in this environment is that the daily context of the user can be connected with the local context, joining current information with educational objectives. For our work, local context is the information about persons, relations, places, and resources in the specific location. This information can be understood by P3A and ESA.

Pervasive Computing is the scope of ISAM project ${ }^{1,16,17}$ which has been developed by our research group. ISAM explores a programming and communication model allowing the implementation of several of these mobile application types. To develop mobile wide-area applications, we consider that mobile hosts must use the existing and accessible fixed network infrastructure, and they have to take advantage of the environment such as the Internet. The ISAM application model considers that "the computer" is the whole network. The computing environment (data, device, code, service, resource) is spread in composed cells. Users can move around, having both their applications and virtual environment following them. The adaptive behavior of the application and the decisions of runtime management are defined by the current context where the application's 
components are inserted. The ISAM project is a free software and could be accessed at http://www.inf.ufrgs.br/ isam.

Several projects were started to investigate the use of web technology, adaptative technical learning, and access to educational servers. Such systems deal with important management issues related to learning courses content, learner stored information, and learning resources in general.

The Edutella Project ${ }^{8}$ provides an RDF-based metadata infrastructure for P2P (Peer-to-Peer) applications exchange the educational resources. Elena ${ }^{14}$ is an operational learning services network based on the interoperable communication infrastructure named "smart spaces for learning". A personal learning assistant is a component of a smart space for learning, which helps learners to search and select learning services. Personal learning assistants are also able to recommend learning services based on the learner profile. The SeLeNe (Self e-Learning Networks) ${ }^{12}$ project offers advanced services for the discovery, sharing and collaborative creation of learning resources, as well as a personalized access to such resources.

The ISAM environment will be used at the GlobalEdu Project, although other systems that support distributed, mobile, context-aware, adaptative and follow-me applications can be probably used. In our proposal, a pervasive personal pedagogical agent involves the learning application or resources and provides a pervasive vision through an application in ISAM environment.

E-Learning metadata standards constitute formal specifications of the descriptive terms used to semantically annotate educational material of all kinds or learner information. This work follows the model defined by Musa ${ }^{8}$ proposal, that uses both $\mathrm{PAPI}^{10}$ and $\mathrm{LIP}^{7}$ standards, learning styles and cognitive styles. These information are extremely important for this work as these styles are the keystone for learning adaptation. More than, this work use a context learning information (see 3.1.3 section).

E-Learning applications are based on the transmission of learning content across various computing environments and platforms; hence, what has to be specified is a structure unit suitable for this interoperation. This "learning unit" is called a Learning Object (LO). From the variety of the eLearning standards proposed from time to time, IEEE LOM standards ${ }^{16}$ specifies nine categories for over 70 metadata elements associated with LOs. This work using five category of the IEEE LOM standards: general, life cycle, technical, educational and rights (see 3.1.2 section). For our propose, these category are interesting for knowledge modeling. 


\section{GLOBALEDU}

We propose a pervasive learning architecture called GlobalEdu. It is composed of educational service agents (ESA) and a pervasive personal pedagogical agent (P3A). ESAs will support learning applications using the pervasive environment ISAM. Agents will be accessed through P3A, which shall always be with the learner (the agent moves to devices that the learner is using - desktop, cellular phone, handhelds, etc), assisting the process of global education through the identification and adaptation of the resources in agreement with the learner's educational model composed of the learner model, learner context and learning object model. Figure 1 presents the GlobalEdu architecture.

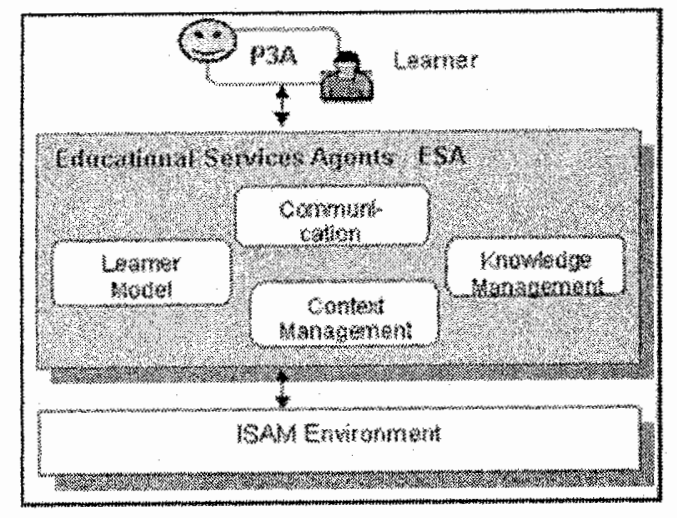

Figure 1. GlobalEdu architecture

\subsection{Pervasive Personal Pedagogical Agent (P3A)}

The P3A is a pervasive tutor, which is always present, accompanying the learner in the devices used. Its characteristics are: (1) Mobility to the devices accessed by the learner; (2) Communication with the ESA in the pervasive environment; (3) Control of disconnection operating normally in the accompanying of the learner, despite some access restrictions to the ESAs and within the capabilities of the device; (4) Suggestion of access to context information related to the location of the learner (local context) with learning goals. P3A contains local agents (micro-agents) responsible for assisting in the process of educational adaptation. It presents two basic types of activities: a cognitive structure based on beliefs and a reactive structure based on events. The beliefs, considered the knowledge of the learner's 
current state, are handled by the local agents Learner Model, Knowledge and Context and used by the local agent Adaptation in the composition of the production rules, being this formalism used for the reasoning of P3A. Events constitute the stimuli necessary for the reaction to the specific transformations seen during processing, characterized by the messages exchanged internally by the local agents $\mathrm{P} 3 \mathrm{~A}$ and between them and the ESAs. The tasks of Local P3A agents are:

\subsubsection{Learner Model}

It is responsible for handling the information about the tutored learner, represented by metadata, assisting the educational adaptation. The metadata Learner Model in this work follows the model suggested by Musa (2004), and some other information. It is assisted by the ESA Learner Model. The category Personal Information contains basic information about the learner so as to identify the learner in the system. Preferences reports the preferences of the learner. The Learner Relations reports the relationships of the learner with other system users. Security deals with security credentials such as: passwords, public and private cryptography keys, used by the learner to access the system. $Q C L$ stands out for Qualifications, Certificates, and Licenses. It contains the learner's experiences regarding certain themes. Goal contains the learner's goals. Learner's abilities, experiences and acquired knowledge are in the category Competency. The category Pedagogical Approach comprehends elements from cognitive and learning styles. The cognitive style is an individual aspect that describes the way in which the person usually approaches or responds to a study task, characterizing a predominance in the access to information. Learning style is a collection of individual abilities and preferences that affect the way the person perceives, searches for and processes information.

The information of Relations and Competence were included into this work so as to meet the competencies of P3A in the pervasive environment, besides the information referring to the logic contexts (see 3.1.3 section) accessed by the learner.

\subsubsection{Knowledge}

It is responsible for managing the Learning Objects (LO) according to the learner model and is supported by the context. It uses standards defined in IEEE/LTSC LOM and is assisted by the ESA Knowledge Management. This work is using five category. The category General provides general information that describe the object. Life Cycle has information that describe the characteristics related to the history and current state of the objects and 
all of those that have affected it during its evolution. The Technical has the technical requirements and characteristics of the object. An important category is Educational, which is responsible for grouping the educational and pedagogical characteristics of the learning object, which are also fundamental for the adaptation process. The Rights has the copyright announcement and the conditions of use of the learning object.

\subsubsection{Context}

It manages information about the Context (logical and physical). The logical context corresponds to the information about people, events, resources, places and spaces. This information is transferred by the ESA Context Management. The physical context corresponds to the context of interest of the educational resources accessed by the learner, such as network, location, device, educational resource and presence of other P3As in the environment. This information composes the Learner Context. The elements that compose the physical context are managed by the Control local agent of P3A and sent to the module local context for the composition of the metadata of Context, according described at table 1. The information about logical and physical context will be used by the module of Adaptation of P3A for the personalization of the educational process and will compose the metadata that represent the Learner Context Model. This way, it will be possible to infer any alteration in the learner model based on the history of learner contexts.

Table 1. Context Information Model

\begin{tabular}{l|l}
\hline FisicalContextInformation & Role \\
& Network \\
& Location \\
& EducationalResource \\
& Device \\
& OtherP3Aldentification \\
\hline LogicalContextInformation & File \\
\hline
\end{tabular}

The information referring to the presence of other P3As in the context in which the learner is currently presupposes the existence of other learners with the same goals, competencies and preferences whose information the learner may access for contact or not. This information can also be used for the creation of learner groups within the same context. 


\subsubsection{Adaptation}

It characterizes and generates educational information, according to the needs and context in which the learner is, defining the most suitable pedagogical model. It is composed of pedagogical goals and strategies which a LO tends to attend. The pedagogical strategies to be used follow the Learning Styles proposed by Felder-Silverman ${ }^{5}$. The pedagogical goals of a LO are the competencies to be developed through the study of the object. Also, through the information referring to the logical context, adaptation is responsible for linking the information of the local where the learner is with the learner's educational goals. The ECA rules have used for adaptation propose.

\subsubsection{Control and Communication}

Control is assisted by the ESA Context Management and is responsible for executing the local procedures to support the physical mobility of the P3A among the several devices used by the learner, as well as perceive the changes of the context elements that integrate the different locations of the learner carrying the same device, characterizing the logical mobility.

Communication manages the exchange of external messages sent and received the ESAs of the environment. Upon receiving a message, it is evaluated and directed to the local destination agents.

\subsection{Educational Service Agents - ESA}

The ESA processes educational services in the context of ISAM pervasive architecture and support the P3A. It requests information or the processing of a certain action from the ESAs, as showed above. The ESAs of GlobalEdu are: (1) Learner Model - it manages the Learner model repository informing and processing information according to the learner tutored by P3A. It establishes the way in which data will be analyzed and information regarding the learner defined; (2) Knowledge Management - it manages the Knowledge Server composed of a repository of learning objects with metadata that describe them; (3) Context Management - it manages context characteristics transmitted by pervasive architecture and by P3A and identifies and manages information regarding the local context in which the learner is; (4) Communication - it manages the external exchange of messages sent and received by the P3A or the environment to the ESAs. Upon receiving a message, it is evaluated and directed to the destination agents. 
All this kind of behavior can be implemented through the ECA rules in order to notify the P3A every time some relevant data is update ou inserted in the repositories.

\section{CONCLUSIONS}

This proposal contributed by the using the educational agents and pervasive personal pedagogical agent to implement and improve the pervasive learning proposal. Moreover, the ISAM project is extremely interesting because it supports a stabilized pervasive environment. Differently from other researches ${ }^{2-4,14,12}$ the proposal supports the execution of context-aware, distributed, mobile and adaptative educational applications. Our proposal supports global communication and continuous computing. Currently, we are implementing the GlobalEdu proposal and integrated in ISAM environment, aiming the validation of the proposed architecture. It will be based on the creation of the logical (metadata information and LO content) physical environment for pervasive learning at Unilasalle, Ufrgs and Unisinos Universities. This activity will encompass the organization of the physical support to execute pervasive learning applications around the campuses. With this, it will be possible for users to change of context keeping continuous computing.

\section{REFERENCES}

1. Augustin, I; et al. ISAM, joining context-awareness and mobility to building pervasive applications. I. Mahgoub and M. Ilyas Ed. Florida. CRC Press. (book chapter published at April, 2004).

2. Chen, Harry; Finin, Tim; Joshi, Anupam. Semantic Web in a Pervasive ContextAware Architecture. Artificial inteligente in Mobile System. In UBICOMP, 2003. October, Seattle, 2003

3. Dagger, D.; Wade, V.; Conlan, O. Towards "anytime, anywhere" Learning: The Role and Realization of Dynamic Terminal Personalization in Adaptive eLearning. In Ed-Media 2003, World Conference on Educational Multimedia, Hypermedia and Telecommunication. Hawai, 2003.

4. Dolog, P.; et al., M. Personalization in Distributed e-Learning Environments. WWW2004, May, New York, 2004. ACM 1-58113-912-8/04/0005

5. Felder, R.M and L.K. Silverman. (1998) Learning and Teaching Styles in Engineering Education, In Engineering Education, 78(7), 674, 1988.

6. IEEE/LTSC Learning Technology standards committee: http://tsc.icee.org 
7. LIP. Learner Information Package Specification 1.0. 2001. Disponivel em: $<\mathrm{http}: / /$ www.imsglobal.org/metadata/index.cfm>. Acesso em Mar. 10, 2005.

8. Musa, D.; Palazzo, J. M.O.; (2004) Sharing Learner Information through a Web services based Learning Architecture. In: WEB INFORMATION SYSTEMS MODELING, WISM, 2004, Riga, Latvia. CAiSE Workshops, Riga, 2004. p.122-131.

9. Ogata, Hiroaki, Yano, Yoneo. How Ubiquitous Computing can support language learning. Proc. of KEST, 2003, p.1-6.

10. PAPI. IEEE P1484.2/d7, 2001. Draft standard for learning technology. Public and Private information para learners. Disponível em: $<$ http://www.edutool.com/papi/>.

11. Roschelle, J.Roy Peã. A walk on the WILD side: How wireless handhelds may change computer-supported collaborative learning. International Conference on Computer-Supported Collaborative Learning (CSCL-02), Boulder, Colorado, January 7-11, 2002.

12. SELENE. Self e-Learning Networks. 2004. Disponível em: http://www.des.bbk.ac.uk/selene/

13. Satyanarayanan, M. Pervasive Computing: Vision and Challenges. IEEE Personal Comunications, New York, v.4, n.8, Aug. 2001.

14. Simon, B.; et al.. Elena: A Mediation Infrastructure for Educational Services. Proc. of WWW Conference. Budapest, Hungary, May 2003.

15. Tatar, D., Jeremy Roschelle, Phil Vahey, William R. Penuel. Handhelds go to school: Lessons Learned. SRI International. Journal Computer, september, 2003, 30-37

16. Yamin, A. C.; et al.. ISAM: a Pervasive View in Distributed Mobile Computing. IFIP/IEEE Network Control and Engineering (NET-CON'2002), 2002, Paris. New York: IEEE Press, 2002.

17. Yamin, A. C.; et al. A Framework for Exploiting Adaptation in High Heterogeneous Distributed Processing. XIV Symposium on Computer Architecture and High Performance Computing (SBAC-PAD), 2002, Vitória. New York: IEEE Press, 2002 